\title{
Mutational analysis of the GNA11, MMP27, FGD1, TRRAP and GRM3 genes in thyroid cancer
}

\author{
AVANIYAPURAM KANNAN MURUGAN, CHONGFEI YANG and MINGZHAO XING \\ Laboratory for Cellular and Molecular Thyroid Research, Division of Endocrinology and Metabolism, \\ The Johns Hopkins University School of Medicine, Baltimore, MD 21217, USA
}

Received December 14, 2012; Accepted April 30, 2013

DOI: $10.3892 / \mathrm{ol} .2013 .1391$

\begin{abstract}
Frequent somatic mutations in the GNA11, matrix metalloproteinase (MMP)27, FGD1, TRRAP and GRM3 genes have been reported in various types of human cancer, but whether these genes are mutated in thyroid cancer is not known. In the present study, a mutational analysis of these genes was performed in thyroid cancer cell lines and thyroid cancer samples. No GNA11 mutations were identified in the papillary thyroid cancer (PTC), follicular thyroid cancer (FTC) and anaplastic thyroid cancer (ATC) samples. Additionally, no mutations were identified in the MMP27 gene, although three synonymous [C351T (N117N), C1089T (S363S) and G1227A (G409G)] single nucleotide polymorphisms (SNPs) were observed infrequently in ATC. No mutations were detected in the FGDl gene, but two infrequent synonymous [T2091C (T697T) and A2136G (P712P)] SNPs were observed in PTC. Furthermore, no mutations were identified in TRRAP and GRM3, although a frequent synonymous SNP [G1323A (T441T)] and infrequent non-synonymous SNP [G1424A (G475D)] of GRM3 were observed in PTC. No mutation of these genes was observed in 12 cell lines derived from various types of thyroid cancer. The present study reports for the first time the mutational status of the GNA11, MMP27, FGD1, TRRAP and GRM3 genes in thyroid cancer. No mutations were identified in these genes in the various types and cell lines of thyroid cancer. Therefore, unlike in other types of cancer, mutations in these genes are absent or uncommon in thyroid cancer.
\end{abstract}

\section{Introduction}

Thyroid cancer is the most common endocrine malignancy with a high incidence in numerous regions of the world $(1,2)$.

Correspondence to: Professor Michael Mingzhao Xing, Division of Endocrinology and Metabolism, The Johns Hopkins University School of Medicine, 1830 East Monument Street, Suite 333, Baltimore, MD 21287, USA

E-mail: mxing1@jhmi.edu

Key words: thyroid cancer, mutation, GNA11, MMP27, FGD1, TRRAP, GRM3
Thyroid cancer is histologically classified into papillary thyroid cancer (PTC), follicular thyroid cancer (FTC) and anaplastic thyroid cancer (ATC). Thyroid cancers frequently harbor activating mutations in the mitogen-activated protein kinase (MAPK) and phosphatidylinositol 3-kinase (PI3K)/Akt signaling pathways, as represented by $R E T / P T C, R A S$ and $B R A F$ mutations in the former and by PIK3CA and PTEN mutations in the latter $(3,4)$. As a significant mechanism for the tumorigenesis of thyroid cancer, aberrant activation of these two important signaling pathways by such mutations causes uncontrolled cell division, proliferation and survival, leading to malignancy.

High-frequency somatic mutations of the GNA11, matrix metalloproteinase (MMP)27, TRRAP and GRM3 genes have been reported in uveal melanomas and melanomas with various incidences (5-8). We previously demonstrated that $F G D 1$ was normally maintained, hypomethylated and overexpressed by $B R A F$ (V600E) in thyroid cancer cells (9). GNA11 activates the MAPK signaling pathway. Particularly frequent somatic mutations of the GNA11 gene at codon 209 in exon 5 and codon 183 in exon 4, resulting in mutant GNA11 ${ }^{\mathrm{Q} 209 \mathrm{~L}}$ and GNA11 ${ }^{\mathrm{R} 183 \mathrm{C}}$, respectively, have been reported in uveal melanoma and blue nevi (5). The GNA11 gene encodes a G-protein $\alpha$-subunit (Go11) that mediates signals from G-protein-coupled receptors (GPCRs) to the MAPK pathway. The normal amino acid, glutamine, encoded by codon 209 of the GNA11 gene, lies within the RAS-like domain of GNA11 (corresponding to residue 61 of Ras) and is essential for GTP hydrolysis. In members of the RAS family, mutations at this site and at codon 12 cause the loss of GTPase activity with constitutive activation of Ras. The GNA11 $1^{\mathrm{Q} 209 \mathrm{~L}}$ and GNA11 ${ }^{\mathrm{R} 183 \mathrm{C}}$ mutants have been demonstrated to be able to transform 3T3 cells and form tumors in immunocompromised mice (5). MMPs are proteolytic enzymes that degrade components of the extracellular matrix and basement membranes. MMP abnormalities have been associated with the metastasis of various types of cancer $(6,10-12)$. In particular, mutations of the $M M P 27$ gene have been observed in melanoma. The majority of the mutations in this gene have been identified in exons 1, 2, 3, 8 and 9 (6). FGD1 gene mutations have been reported in Aarskog-Scott syndrome (AAS), or facio-digito-genital dysplasia (13). At present, 20 different FGD1 gene mutations have been reported in this syndrome (13). FGD1 is a Dbl family member that has been 
Table I. Primer sets used for PCR amplification of the MMP27 and FGD1 genes.

\begin{tabular}{cll}
\hline Exon & \multicolumn{1}{c}{ Forward } & \multicolumn{1}{c}{ Reverse } \\
\hline$M M P 27$ & & \\
$1-1$ & GCCAATTCATGCCACGTCTCAC & GAAAATATGCAACTGGCTCAGG \\
$1-2$ & GAACCGGCTTCAGCTGAAGAAAG & CACATTCCTGCAAAAGAGTCCTG \\
$2-1$ & CCTGAGATGGAGATTTCTCTC & GGATTGACAGTGACTGGAAAAC \\
$2-2$ & TCTTTTGGTCAGGCATATCTC & ATCATG AAGACACCCAGGTGTG \\
$2-3$ & CTCAACCAGT TCTACTCTCTTG & GTATGGCTACACCCTCCCT \\
$2-4$ & TGAGATCATG AAGACACCCAGG & CATTCAATTGACTGAGCACTTC \\
$F G D 1$ & & \\
1 & GGCTTGAGTCTCTGCAGTG & AACAAGAACCCGCTCCCAGTAC \\
2 & AGTCCTAACTTTAACCCCAGTC & CTGGCTAACTTCTCCCCTCCTC \\
3 & TTCACCATGTTAGCCAGGCTC & GTATGAGCTTGACTGAGAGGC \\
4 & AGCCTGGGACAGGAAGGGATA & AAAGGCGCTTCCAGGTTCTCC \\
5 & TATTAGGCTTAGAGTGGCATG & ACTGCCTCCTTGAAACGCACC \\
6 & TCAGTCTCAAGACCAATGCTG & GAAGTCTTGTGTACACCTCTG \\
7 & ACTGAGATGAAAGGTATCTGC & TCAGATCTGGCTGCAGATGCC \\
8 & AAGCTGGAAGGAGCAGACTTG & AGAGCTATTAGTGTGGAGAAG \\
9 & AGGCAGAGGTTGTGGTGAGCC & GTGCCAGCCTCCTGTCAGATG \\
10 & ATCTGACAGGAGGCTGGCACC & AGTACCAGGTCACTATGTGTG \\
11 & CAGTAAAGCTTCAGGGCAAG & AGCCAGCATCTTTGTCCTC \\
12 & TGTGTGTGTATGTGTGCAGAG & TCTCTGGGCCTGGAATGCCTC \\
13 & AGGTGAAGAAGAGGGTCAAGC & ACCATTCTGGTTAGCTGTGAG \\
14 & CTAGGGTATACGAAGGTGAGGC & TAAAGGTCAGGTGGGCATTTGG \\
16 & ATGATAATCCAAGCGTTGGAG & AGGGTACCCACTCTGCAGTGG \\
18 & TGCTGTGGGAGTTGGTATGCTG & TGCTGTGGGAGTTGGTATGCTG \\
\hline & GCAAAACCAGTTAGAAGCTGGG & TCTTCCAAGGCTCACCTTATC \\
& TTCAAGTATTGACTGAGCTGGG \\
\hline
\end{tabular}

Primers are represented as $5^{\prime}>3^{\prime}$. MMP, matrix metalloproteinase.

shown to function as a CDC42-specific guanine nucleotide exchange factor (GEF). It has also been demonstrated that FGD1 expression is sufficient to cause tumorigenic transformation of NIH3T3 fibroblasts (14). Two studies from the same group reported that the TRRAP gene was recurrently mutated and clustered in one amino acid position S722F (7). Furthermore, a frequent mutation of the GRM3 gene has been reported, and the authors also noted that the mutant selectively regulated the phosphorylation of MEK in the activation of the MAPK signaling pathway, leading to the anchorage-independent growth and migration of cells (8). The mutation status in the GNA11, MMP27, FGD1, TRRAP and GRM3 genes has not been studied in thyroid cancer. The present study was conducted to investigate the mutational status of these genes in thyroid cancer.

\section{Materials and methods}

Cell lines, tumor samples and DNA extraction. A total of 89 samples, consisting of 12 thyroid cancer cell lines and 77 thyroid tumor samples were used for the mutational analysis of the GNA11 gene. For the MMP27 mutational analysis, 29 samples consisting of 12 thyroid cancer cell lines and 17 ATC samples were used. The FGD1, TRRAP and GRM3 genes were analyzed in 28 samples, including 12 thyroid cancer cell lines and 16 PTC samples. The thyroid cancer cell lines and tumor samples were used as described previously and with institutional review board (IRB; The Johns Hopkins University School of Medicine, Baltimore, MD, USA) approval (15). The cell lines were authenticated as described previously (16). With the exception of the FTC133 cells cultured in Dulbecco's modified Eagle's medium (DMEM)/Ham's F-12 medium, all tumor cell lines were cultured in RPMI-1640 medium supplemented with $10 \%$ fetal bovine serum (FBS), streptomycin $(100 \mu \mathrm{g} / \mathrm{ml})$, penicillin $(100 \mathrm{U} / \mathrm{ml})$ and $2 \mathrm{mM}$ glutamine. Genomic DNA from the cell lines and tumors was isolated by standard phenolchloroform extraction using MaXtract high-density gel tubes followed by ethanol precipitation procedures (Qiagen, Valencia, CA, USA) (15).

PCR amplification and sequencing of the GNA11, MMP27, FGD1, TRRAP and GRM3 genes. The primer sequences and PCR conditions for the amplification of exons 4 and 5 
Table II. Mutations of the MMP27, FGD1 and GRM3 genes in thyroid cancers.

\begin{tabular}{lcrcccc}
\hline S. No. & Gene & Exon & Nucleotide & Codon & Amino acid & Type of mutation \\
\hline ATC\# 12 & MMP27 & 3 & C351T & AAC-AAT & N117N & Silent \\
ATC\# 19 & MMP27 & 8 & C1089T & TCC-TCT & S363S & Silent \\
ATC\# N3 & MMP27 & 9 & G1227A & GGG-GGA & G409G & Silent \\
PTC\# 4 & FGD1 & 14 & T2091C & ACT-ACC & T697T & Silent \\
PTC\# 52 & FGD1 & 14 & A2136G & CCA-CCG & P712P & Silent \\
PTC\# 6 & GRM3 & 3 & G1323A & ACG-ACA & T441T & Silent \\
PTC\# 46 & GRM3 & 3 & G1424A & GGT-GAT & G475D & Missense \\
\hline
\end{tabular}

MMP, matrix metalloproteinase.

of the GNA11 gene and exons 1,2,3,8 and 9 of the MMP27 gene were as described previously $(5,6)$. For the mutational analysis of the $M M P 27$ gene, in addition to the above primers, additional primers were used to shorten the amplicon size. These primers are shown in Table I. The PCR conditions for the amplification of the $M M P 27$ with the additional primers were as follows: One cycle of $94^{\circ} \mathrm{C}$ for $3 \mathrm{~min}$; ten cycles of $94^{\circ} \mathrm{C}$ for $30 \mathrm{sec}, 67^{\circ} \mathrm{C}$ for $30 \mathrm{sec}$ with a $1^{\circ} \mathrm{C}$ reduction for each cycle and $72^{\circ} \mathrm{C}$ for $30 \mathrm{sec}$; thirty two cycles of $94^{\circ} \mathrm{C}$ for $30 \mathrm{sec}$, $57^{\circ} \mathrm{C}$ for $30 \mathrm{sec}$ and $72^{\circ} \mathrm{C}$ for $30 \mathrm{sec}$; followed by $72^{\circ} \mathrm{C}$ for $7 \mathrm{~min}$ as a final extension; and $4^{\circ} \mathrm{C}$ as the storage temperature. The primer sequences for the amplification of the FGDl gene are shown in Table I. The PCR reaction conditions for the FGDI amplification were as follows: After an initial denaturation at $94^{\circ} \mathrm{C}$ for $2 \mathrm{~min}$, the amplification was performed at $94^{\circ} \mathrm{C}$ for $1 \mathrm{~min}$, followed by annealing temperatures (exons 11,14 and $18,55^{\circ} \mathrm{C}$; exons 1,2 and $16,57^{\circ} \mathrm{C}$; exons $5,6,7,8$, 13 and $17,58^{\circ} \mathrm{C}$; exons 3,12 and $15,60^{\circ} \mathrm{C}$; and exons 4,9 and $10,62^{\circ} \mathrm{C}$ ) for $1 \mathrm{~min}$ for 35 cycles, with a final extension at $72^{\circ} \mathrm{C}$ for $7 \mathrm{~min}$. The primer sequences and PCR conditions for the amplification of exon 1 of the TRRAP gene and exons 1, 2, 3, 4 and 5 of the GRM3 gene were as described previously $(7,8)$. The PCR products were directly sequenced using a BigDye Terminator v3.1 Cycle Sequencing ready reaction kit (Applied Biosystems, Foster City, CA, USA). These exons were investigated as they harbored the majority of the reported mutations in these genes in human cancers. The GenBank accession numbers were NM_002067.2 (GNA11), NM_022122 (MMP27), NM_004463 (FGD1), NM_003496 (TRRAP) and NM_000840 (GRM3).

\section{Results}

The strategy of the present study was to investigate the gene exons that were the most likely to carry mutations. In particular, exons 4 and 5 of the GNA11 gene were examined for mutations since all of the known GNA11 mutations have been reported in codons 209 and 183 of these exons. Exons 1, 2, 3, 8 and 9 of the MMP27 gene were selected for sequencing as they have been shown to carry somatic mutations in melanomas. All the exons of the FGDl gene were analyzed for mutation, as mutations in this gene have never been reported in human cancers. Exon 1 of the TRRAP gene and exons 1,2,3,4 and 5 of the GRM3 gene were analyzed as these exons have also been reported to harbor somatic mutations in melanoma.

The sequencing results showed no mutations in and around the hot spot of codons 209 and 183 in the GNA11 gene in 12 thyroid cancer cell lines and 46 thyroid cancer samples (including 26 FTC and 20 ATC samples). No novel MMP27 somatic mutations were identified in 12 thyroid cancer cell lines and 15 ATC tumor samples. As shown in Fig. 1A and B, an infrequent [1/17 (5.8\%)] $\mathrm{C}>\mathrm{T}$ transition was observed at nucleotide position 351, resulting in a codon change of AAC $>$ AAT and amino acid $\mathrm{N} 117 \mathrm{~N}$ in exon 3 . In exon 8 , an infrequent $[1 / 17$ (5.8\%)] $\mathrm{C}>\mathrm{T}$ transition was observed at nucleotide position 1089, resulting in a codon change of TCC $>$ TCT and amino acid S363S. In exon 9, a frequent [7/17 (41.2\%)] G>A transition at nucleotide position 1227 was also observed, resulting in a codon change of GGG>GGA and amino acid G409G. The two N117N and S363S mutations were rare and novel silent mutations that have not been previously reported in the SNP database.

No FGD1 mutations were identified in 12 thyroid cancer cell lines. However, as illustrated in Fig. 1C and D, an infrequent [1/16 (6.3\%)] $\mathrm{T}>\mathrm{C}$ mutation was observed at nucleotide position 2091, resulting in a codon change of ACT $>$ ACC and amino acid T697T. An infrequent [1/16 (6.3\%)] $A>G$ was also observed at nucleotide position 2136 , resulting in a codon change of CCA $>C C G$ and amino acid P712P. These silent T697T (rs12011120) and P712P (rs1126744) mutations were rare SNPs that have been reported in the SNP database (http://www.ncbi.nlm.nih.gov/projects/SNP/).

Mutations were not identified in the TRRAP gene in 12 thyroid cancer cell lines and 16 PTC tumor samples. No GRM3 mutations were detected in 12 thyroid cancer cell lines. A $\mathrm{G}>\mathrm{A}$ mutation was observed at nucleotide position 1323, resulting in a codon change of $\mathrm{ACG}>\mathrm{ACA}$ and amino acid T441T in all 12 thyroid cancer cell lines and 16 PTC samples. An infrequent [1/16 (6.3\%)] G>A mutation was also observed at nucleotide position 1424 resulting in a codon change of GGT>GAT and amino acid G475D (Fig. 1E). The T441T mutation was a novel synonymous SNP that has not previously been reported in the SNP database. G475D (rs17161026) was a non-synonymous SNP that has previously been reported in the SNP database (http://www.ncbi.nlm.nih.gov/projects/SNP/). Fig. 1 shows the mutations identified in the present study, and a summary of the results is presented in Table II. 
A



Figure 1. Detection of MMP27, FGD1 and GRM3 mutations. (A) Sequencing results are shown with a representative sense sequence profile of SNPs (N117N, S363S and G409G) detected in exons 3, 8 and 9 of the MMP27 gene. Arrows indicate mutated nucleotides. (B) Schematic diagram of domains of the MMP27 protein showing three SNPs (N117N, S363S and G409G) identified in thyroid cancer. (C) Sequencing results are shown with a representative sense sequence profile of SNPs (T697T and P712P) detected in exon 14 of the FGD1 gene. Arrows indicate mutated nucleotides. (D) Schematic diagram of domains of the FGD1 protein showing two SNPs (T697T and P712P) identified in thyroid cancer. (E) Sequencing results are shown with a representative sense sequence profile of two SNPs (T441T and G475D) detected in exon 3 of the GRM3 gene. Arrows indicate mutated nucleotides. The nucleotide, amino acid alteration and tumor number are indicated above the arrows. Nucleotide numbers refer to the position within the coding sequences, where position 1 corresponds to the first position of the initiation codon. All the samples were sequenced in two repeated examinations with independent PCR by forward and reverse primers. MMP, matrix metalloproteinase; SNPs, single nucleotide polymorphisms.

\section{Discussion}

In the present study, several genes were analyzed for the first time for possible mutations in thyroid cancer. GNA11 mutations were analyzed in all types of thyroid cancer (PTC, FTC and ATC) as they have been frequently identified in uveal melanoma and are known to activate the MAPK signaling pathway (5), which is one of the most deregulated signaling pathways in thyroid cancer (3). However, no mutations were detected in and around codons 209 and 183. These two hot spot codons were selectively analyzed as GNA11 mutations have consistently been identified only in these two residues (5). The MMPs are proteolytic enzymes that degrade the components of the extracellular matrix and basement membranes, which are associated with cancer metastasis $(6,10-12)$. ATC is the most aggressive type of thyroid cancer that is often associated with deadly metastasis (17). Therefore ATC was particularly analyzed for the mutation of the MMP27 gene. Two MMPs have been reported to occasionally be mutated in melanomas. The MMP27 gene was analyzed for mutation in ATC in the present study as we had already previously analyzed the second gene, $M M P 8$, in thyroid cancer (18). Three uncommon 
mutations were identified; C351T resulting in N117N, C1089T resulting in S363S and G1227A resulting in G409G silent mutations. The silent mutations are unlikely to be involved in thyroid carcinogenesis as these mutations do not change the basic amino acids.

We previously revealed that $F G D 1$ was normally maintained, hypomethylated and overexpressed by BRAF (V600E) in thyroid cancer cells and in turn observed that it was hypermethylated after ShRNA-mediated knockdown of BRAF (V600E) in thyroid cancer cell lines (9). Based on these findings and the high transforming and invasive potential of the FGDl gene (4), we considered there to be a high possibility of identifying oncogenic mutations in FGD1. All 18 exons of the gene were sequenced to be analyzed for mutations, but only two silent mutations (T697T and P712P) were detected. These mutations are unlikely to have a significant role in PTC. No somatic missense mutations were identified in the FGDl gene.

The TRRAP gene has been reported to be mutated in a particular codon, S722F (7). As GRM3 activates the MAPK signaling pathway (8), the present study investigated whether GRM3 is mutated in PTC samples, since the majority of PTCs harbor genetic deregulation in the MAPK signaling pathway. No mutations were detected in TRRAP, while two SNPs (T441T and G475D) were identified in the GRM3 gene. This suggests that TRRAP and GRM3 may not have important roles in the pathogenesis of this type of thyroid cancer.

In conclusion, the present findings suggested that genetic alterations in the GNA11, MMP27, FGD1, TRRAP and GRM3 genes may not be significant in the tumorigenesis of thyroid cancer. It is not surprising that mutations in these genes are not common in thyroid cancer since a number of the upstream effectors involved in cellular transformation, growth and metastasis, including EGFR, RET/PTC, ALK, RAS, BRAF, PTEN, PIK3CA, PIK3CB and PDK1, are commonly genetically altered via mutations or genetic amplifications that are able to independently activate the MAPK or PI3K/Akt pathways in thyroid cancer $(3,4,16,18,19)$.

\section{Acknowledgements}

The authors would like to thank Drs N.E. Heldin, K.B. Ain, N. Onoda, M. Santoro, D. Wynford Thomas, G. Brabant, A.P. Dackiw, R Schweppe and B Haugen for kindly providing access to the cell lines used in the present study. The study was supported by NIH R01 grant CA 134225.

\section{References}

1. Leenhardt L, Grosclaude P and Chérié-Challine L; Thyroid Cancer Committee: Increased incidence of thyroid carcinoma in France: a true epidemic or thyroid nodule management effects? Report from the French Thyroid Cancer Committee. Thyroid 14: 1056-1060, 2004.

2. Sprague BL, Warren Andersen S and Trentham-Dietz A: Thyroid cancer incidence and socioeconomic indicators of health care access. Cancer Causes Control 19: 585-593, 2008.

3. Xing M: BRAF mutation in thyroid cancer. Endocr Relat Cancer 12: 245-262, 2005.

4. Liu Z, Hou P, Ji M, et al: Highly prevalent genetic alterations in receptor tyrosine kinases and phosphatidylinositol 3-kinase/akt and mitogen-activated protein kinase pathways in anaplastic and follicular thyroid cancers. J Clin Endocrinol Metab 93: 3106-3116, 2008.

5. Van Raamsdonk CD, Griewank KG, Crosby MB, et al: Mutations in GNA11 in uveal melanoma. N Engl J Med 363: 2191-2199, 2010.

6. Palavalli LH, Prickett TD, Wunderlich JR, et al: Analysis of the matrix metalloproteinase family reveals that MMP8 is often mutated in melanoma. Nat Genet 41: 518-520, 2009.

7. Wei X, Walia V,Lin JC, et al: Exome sequencing identifies GRIN2A as frequently mutated in melanoma. Nat Genet 43: 442-446, 2011.

8. Prickett TD, Wei X, Cardenas-Navia I, et al: Exon capture analysis of $\mathrm{G}$ protein-coupled receptors identifies activating mutations in GRM3 in melanoma. Nat Genet 43: 1119-1126, 2011.

9. Hou P, Liu D and Xing M: Genome-wide alterations in gene methylation by the BRAF V600E mutation in papillary thyroid cancer cells. Endocr Relat Cancer 18: 687-697, 2011.

10. Brinckerhoff CE and Matrisian LM: Matrix metalloproteinases: a tail of a frog that became a prince. Nat Rev Mol Cell Biol 3 207-214, 2002

11. Egeblad M and Werb Z: New functions for the matrix metalloproteinases in cancer progression. Nat Rev Cancer 2: 161-174, 2002.

12. Freije JM, Balbín M, Pendás AM, Sánchez LM, Puente XS and López-Otín C: Matrix metalloproteinases and tumor progression. Adv Exp Med Biol 532: 91-107, 2003.

13. Orrico A, Galli L, Faivre L, et al: Aarskog-Scott syndrome: clinical update and report of nine novel mutations of the FGD1 gene. Am J Med Genet A 152A: 313-318, 2010.

14. Whitehead IP, Abe K, Gorski JL and Der CJ: CDC42 and FGD1 cause distinct signaling and transforming activities. Mol Cell Biol 18: 4689-4697, 1998

15. Murugan AK, Dong J, Xie J and Xing M: MEK1 mutations, but not ERK2 mutations, occur in melanomas and colon carcinomas, but none in thyroid carcinomas. Cell Cycle 8: 2122-2124, 2009.

16. Murugan AK and Xing M: Anaplastic thyroid cancers harbor novel oncogenic mutations of the ALK gene. Cancer Res 71: 4403-4411, 2011.

17. Neff RL, Farrar WB, Kloos RT and Burman KD: Anaplastic thyroid cancer. Endocrinol Metab Clin North Am 37: 525-538, 2008.

18. Murugan AK, Dong J, Xie J and Xing M: Uncommon GNAQ, MMP8, AKT3, EGFR, and PIK3R1 mutations in thyroid cancers. Endocr Pathol 22: 97-102, 2011.

19. Mitsiades CS, Kotoula V, Poulaki V, et al: Epidermal growth factor receptor as a therapeutic target in human thyroid carcinoma: mutational and functional analysis. J Clin Endocrinol Metab 91: 3662-3666, 2006. 